\title{
A NON-INVASIVE TECHNIQUE FOR CONFIGURING LOW LEVEL RF FEEDBACK LOOPS IN PEP-II *
}

\author{
D. Teytelman ${ }^{\dagger}$, \\ SLAC, Menlo Park, CA 94025, USA
}

\begin{abstract}
The RF system of the PEP-II collider uses two fast feedback loops around each klystron and set of cavities. These loops reduce the impedance of the fundamental mode of the accelerating cavities seen by the beam, and are necessary to reduce the growth rates of longitudinal modes within the RF system bandwidth. Operation of the accelerator at high beam currents is very sensitive to the configuration of the low-level RF feedback loops. There are 7 loop control parameters that strongly influence the stability of the feedback loops and the achieved level of longitudinal impedance reduction. Diagnostic techniques for the analysis of the RF feedback via closed-loop system transfer function measurements will be presented. The model is fit to the measured closed-loop transfer function data and the extracted parameters are then used to calculate optimal tuning and corrections to the loop control elements in the physical channel. These techniques allow fine-tuning of RF feedback with stored beam as well as diagnosis of misconfigured or malfunctioning elements of the system. Results from PEP-II operation will be presented to illustrate the techniques and their applications.
\end{abstract}

\section{INTRODUCTION}

Both electron (HER) and positron (LER) rings of the PEP-II collider operate at high beam loading which leads to cavity detuning frequencies exceeding the ring revolution frequency of $136 \mathrm{kHz}$. In order to reduce the longitudinal coupled-bunch instability growth rates driven of the cavity fundamental impedance two fast feedback loops are applied: the direct loop and the comb loop [1,2]. The direct loop is a proportional feedback channel that compares the vector sum $V_{\text {total }}$ of cavity signals to the complex station reference and applies appropriate drive signal to the klystron to maintain the sum constant. Since the direct loop gain is limited by the overall system group delay, a comb filter is used to provide additional gain at the synchrotron sidebands of the revolution harmonics.

\section{LLRF FEEDBACK CONFIGURATION}

A set of automated configuration procedures has been developed during the system commissioning [3] for optimally configuring the direct and comb loops. These procedures measured the open-loop transfer functions for the

\footnotetext{
* Work supported by U.S. Department of Energy contract DE-AC02$76 \mathrm{SF} 00515$

†dim@slac.stanford.edu
}

direct and comb loops, fitted the loop models to these measured responses and came up with the gain, phase and delay settings. Normally the feedbacks are configured for maximum impedance reduction in which case the overall system has around $4 \mathrm{~dB}$ of gain margin and 35 degrees of phase margin. Since in the open-loop configuration (without impedance control) the instability growth rates exceed controllable limits at relatively moderate beam currents all of these configuration procedures are necessarily performed without beam in the ring at the low klystron output power of $120-200 \mathrm{~kW}$.

However the operating point of the RF system is not fixed - the output power of a station goes from a minimum of $120 \mathrm{~kW}$ without beam to $1.2 \mathrm{MW}$ at the maximum beam loading. Such a change in the klystron output power is accompanied by significant changes in the klystron large and small-signal gains as well as in the group delay and the insertion phase. Several low-bandwidth tracking loops are used to compensate for these changes. However the errors in tracking as well as the uncompensated dynamics changes can shift the loop response between low and high output power conditions. This shift is exhibited in reduced LLRF feedback stability margins, imperfect impedance reduction, and, in some cases, in internal instability and oscillation. In order to alleviate these effects it is desirable to be able to measure feedback loop configuration at high currents and make the necessary corrections to the feedback loop settings. A method described in this paper has been developed in response to such a need. The technique uses a linear model of the RF system and the feedback loops to fit a closed-loop transfer function measured non-invasively at high beam current and extracts the loop control parameters. Comparing the extracted parameters with the model-based optimal settings provides a set of parameter errors that need to be adjusted out for best stability margins and impedance control.

\section{A LINEAR MODEL}

A simplified linear model shown in Fig. 1 is at the heart of this non-invasive diagnostic and configuration method. A set of two or four RF cavities is replaced by an equivalent cavity model that assumes that all cavities are at the same detuning and the cavity voltages are added in-phase - a safe assumption for a properly maintained station. The model consists of the RF cavity and the two fast feedback loops. The responses of the lead compensation, comb filter and the equalizer FIR filter are modeled based on the actual coefficients and the knowledge of the hardware implemen- 


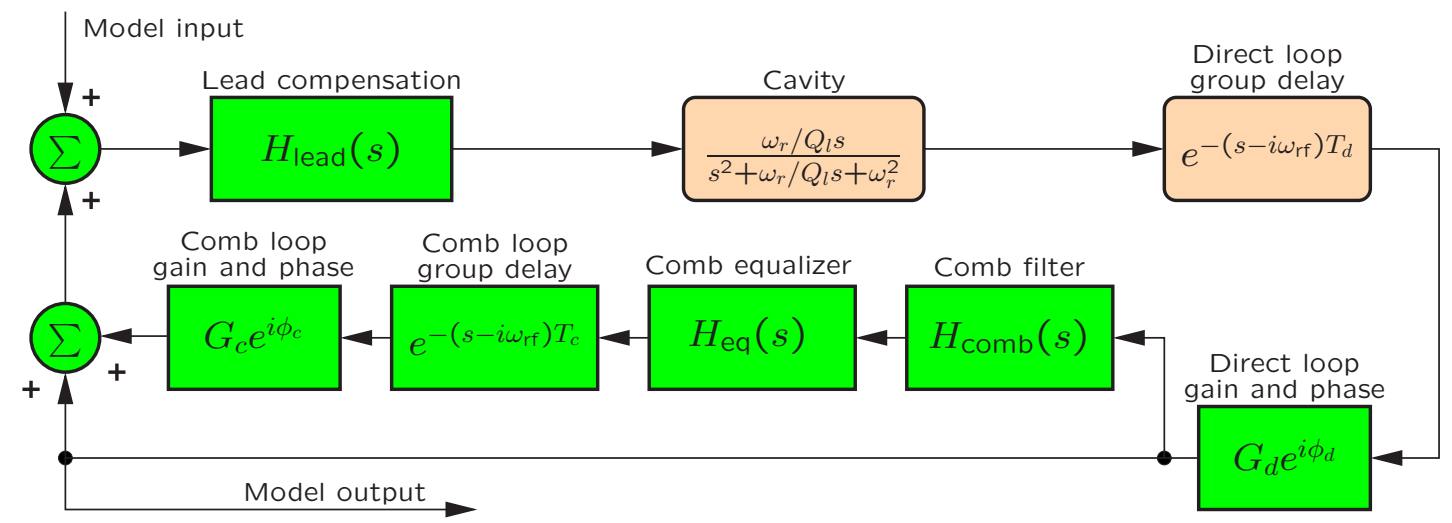

Figure 1: A block diagram of the PEP-II RF linear model

tation. That leaves 8 parameters: cavity resonant frequency $\omega_{r}$, cavity loaded quality factor $Q_{l}$, direct loop gain $G_{d}$, direct loop delay $T_{d}$, direct loop phase shift $\phi_{d}$, comb loop gain $G_{c}$, comb loop delay $T_{c}$, and comb loop phase $\phi_{c}$. Even though the summing sign is positive in the block diagram the feedback is still negative due to the direct loop phase $\phi_{d}$ nominal setting of 180 degrees.

\section{TRANSFER FUNCTION MEASUREMENT}

A closed-loop system transfer function is measured by injecting noise at the reference summing junction and recording the response at the output of the direct loop. Excitation noise is shaped by a low-pass filter to place signal power within the RF system's closed-loop bandwidth. A set of Matlab tools has been developed to measure calibration responses of the digital network analyzers built-in the RF processing (RFP) modules of PEP-II LLRF [3]. Such calibrations help improve the quality of the measured transfer function leading to improved model fitting.

\section{MODEL PARAMETER EXTRACTION}

The overall model transfer function used to fit the measured closed-loop response is given by

$$
H_{\text {meas }}(s)=\frac{L_{\mathrm{dir}}(s)}{1-\left(1+L_{\mathrm{comb}}(s)\right) L_{\mathrm{dir}}(s)}
$$

where $L_{\mathrm{dir}}(s)$ is the open-loop transfer function of the direct loop and $L_{\text {comb }}(s)$ is the open-loop transfer function of the comb loop. According to the system block diagram shown in Fig. 1 these open loop functions are given by

$$
\begin{array}{r}
L_{\mathrm{dir}}(s)=\frac{2 \sigma s}{s^{2}+2 \sigma s+\omega_{r}^{2}} H_{\text {lead }}(s) G_{d} e^{i \phi_{d}-\left(s-i \omega_{\mathrm{rf}}\right) T_{d}} \\
L_{\mathrm{comb}}(s)=H_{\mathrm{comb}}(s) H_{\mathrm{eq}}(s) G_{c} e^{i \phi_{c}-\left(s-i \omega_{\mathrm{rf}}\right) T_{c}}
\end{array}
$$

where $\sigma=\omega_{\mathrm{rf}} /\left(2 Q_{l}\right)$ is the damping time of the cavity.

As mentioned earlier there are 8 individual parameters defining the model configuration. However in model fitting $\omega_{r}$ is determined by the average cavity detuning as re- ported by the tuner position readback and $Q_{l}$ is set to the nominal value based on the cavity design $Q$ and the design coupling factor $\beta_{0}$. This decision is based on the empirical experience showing that the error function versus these two parameters is quite shallow while errors in these variables lead to large errors in the estimation of the other six parameters.

The six-dimensional optimization is then set up with the frequency-weighted error function given by

$$
f(\vec{x})=\sum_{\omega} W(\omega)\left|H_{\text {meas }}(i \omega)-H_{\text {model }}(\vec{x}, i \omega)\right|^{2}
$$

where $\vec{x}$ is a vector of six optimization parameters and $W(\omega)$ is a weighting function used to amplify the highfrequency error components where the system response is rolling off. Such weighting improves high-frequency fitting which is mostly manifested in a more accurate estimate for the direct-loop group-delay $T_{d}$. The summing is performed at the range of frequencies from 475 to $477 \mathrm{MHz}$.

Initial parameters for the optimization vector are empirically preset for good convergence for the transfer functions measured in the configurations within the normal operating range of the HER or the LER. Sometimes the initial point has to be adjusted to achieve convergence in the unusual conditions.

\section{RF SYSTEM TUNING EXAMPLE}

In this example tuning of the LLRF feedback loops without beam will be illustrated. An initial transfer function measurement in Fig. 2 shows clear mistuning exhibited in the asymmetric response and significant peaking at 165 and $290 \mathrm{kHz}$. When the system is so dramatically mistuned the linear model fit is degraded. However the fit is still close enough to indicate the following errors: direct loop gain $2 \mathrm{~dB}$ low, -7 degrees offset in the direct loop phase, comb gain $0.8 \mathrm{~dB}$ high, $100 \mathrm{~ns}$ error in the comb one-turn delay, and a -21 degrees comb phase offset. Since the indicated parameter errors are large in the first tuning step only the comb loop corrections are applied which lower 

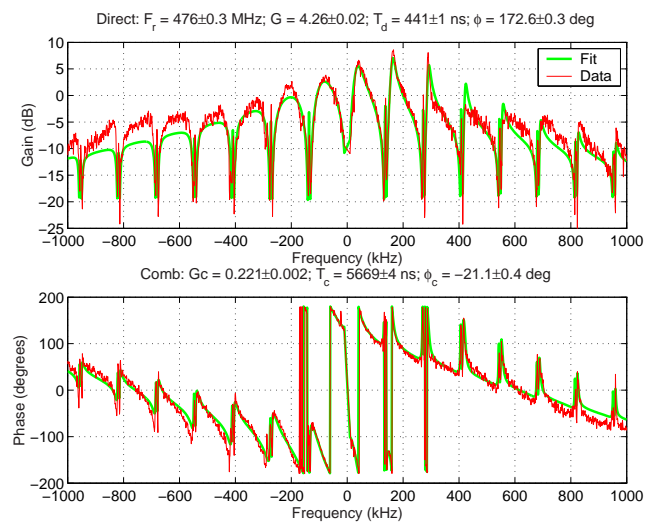

Figure 2: A transfer function measurement and a fit for the badly configured LER RF station 4-5

the gain by $0.8 \mathrm{~dB}$ and move the phase by 21 degrees. Both the measured response and the model fit are significantly improved by this adjustment. Table 1 lists the extracted parameters after each tuning stage as well as the optimal settings. Note that the estimates of the comb phase and delay shift between the first and the second stage inconsistently with the applied adjustments. These shifts are attributed to the model fitting errors in the first stage.

In the second adjustment $G_{d}$ is raised by $1 \mathrm{~dB}, \phi_{d}$ is rotated by 8.6 degrees, $T_{c}$ lowered by $100 \mathrm{~ns}$, and $\phi_{c}$ is rotated by -2.5 degrees. The resultant response and the fit are shown in Fig 3. Note the significantly improved response symmetry around the RF frequency and the absence of the underdamped peaks. The direct loop gain is set $2.6 \mathrm{~dB}$ low on purpose since the klystron gain tracking loop is normally configured with a $2.5 \mathrm{~dB}$ offset without beam. Such an offset allows to better utilize the limited dynamic range of the tracking loop at high beam currents. The error in the comb filter delay is $12.8 \mathrm{~ns}$ which is smaller than a $25 \mathrm{~ns}$ step of the comb delay adjustment.

\section{FREQUENCY TRACKING LOOP CONFIGURATION}

As the RF station tuning example above illustrated the optimal phasing of the direct and comb loops is critical in order to achieve good loop stability as well as maximum impedance reduction. However as the RF cavities are de-

Table 1: Extracted model parameters during station tuning

\begin{tabular}{lcccc} 
Parameter & Stage 1 & Stage 2 & Stage 3 & Optimal \\
\hline$G_{d}, \mathrm{~dB}$ & 12.59 & 13.14 & 14.58 & 17.2 \\
$T_{d}, \mathrm{~ns}$ & 441 & 444 & 442 & $\mathrm{n} / \mathrm{a}$ \\
$\phi_{d}$, degrees & 172.6 & 171.4 & 179.2 & 180 \\
$G_{c}, \mathrm{~dB}$ & -13.15 & -14 & -14 & -14 \\
$T_{c}, \mathrm{~ns}$ & 5669 & 5630 & 5548 & 5561 \\
$\phi_{c}$, degrees & -21.1 & 2.5 & 0.3 & 0
\end{tabular}
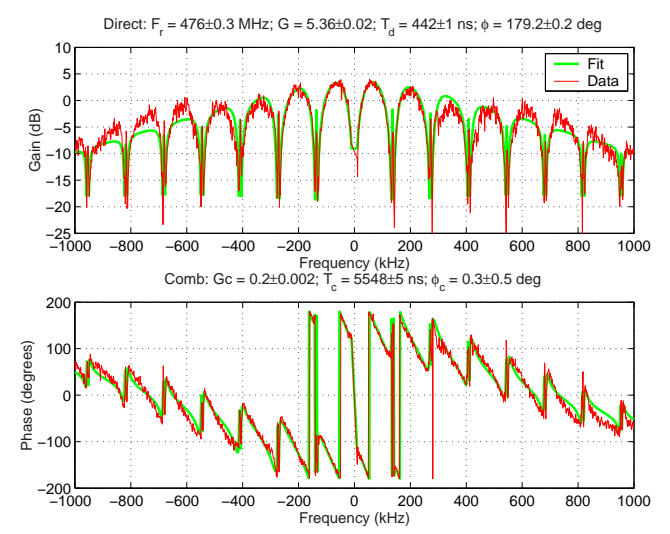

Figure 3: A transfer function measurement and a fit after tuning of the LER RF station 4-5

tuned the phase centering is lost due to the resonator response "sliding" on the group delay slope of the direct loop. In order to restore the phase margins the frequency tracking loop adjusts direct (and comb) loop phase shifters as a function of cavity detuning. From the transfer function measurements and the extracted model we determine two additional parameters - the tracking gains of the direct and comb loops.

\section{CONCLUSIONS}

Precise control of LLRF feedback configurations in PEP-II is necessary for the reliable system operation. Configuring the feedback loops at the nominal station operating powers and cavity detuning frequencies provides such precise and reliable control. The model-based parameter extraction method presented in this paper has been instrumental for performing such configuration verification and RF station tuning. The method has been used almost exclusively for LLRF feedback tuning in PEP-II for the last 3 years and allowed to almost completely eliminate beam downtime due to the misconfigured loop parameters.

\section{REFERENCES}

[1] F. Pedersen, "RF cavity feedback," in B factories: the state of the art in accelerators, detectors and physics: proceedings, pp. 192-207, SLAC, 1992.

[2] P. Corredoura et al., "Low level RF system design for the PEP-II B factory," in Proceedings of the 1995 Particle Accelerator Conference and International Conference on High Energy Accelerators, (Piscataway, NJ, USA), pp. 2672-2674, IEEE, 1996.

[3] P. Corredoura et al., "Commissioning experience with the PEP-II low-level RF system," in 1997 IEEE Particle Accelerator Conference: Proceedings, (Piscataway, NJ, USA), pp. 2359-2361, IEEE, 1998. 